\title{
El emprendimiento en la mujer rural del siglo XXI
}

\section{Entrepreneurship in rural women of the 21st century}

\author{
Alcides Antúnez Sánchez ${ }^{1} \bowtie$ \\ ${ }^{1}$ Máster en Asesoría Jurídica mención Derecho Ambiental por la Facultad de Derecho de la Universidad \\ de Oriente Licenciado en Derecho y Ciencias Penales. Profesor Auxiliar Derecho Ambiental e Internacional. \\ Departamento de Derecho. Facultad de Ciencias Económicas y Sociales. Universidad de Granma. \\ República de Cuba.
}

Correo electrónico: aantunez@udg.co.cu; antunez63@nauta.cu, antunez1963@gmail.com

Recibido: 10 de septiembre del 2018 Aprobado: 12 de diciembre 2018 Disponible en línea: 5 de enero del 2019

Cómo citar este artículo: Alcides Antúnez Sánchez. El emprendimiento en la mujer rural del siglo XXI. DIXI 28. Julio 2018. doi: https://doi.org/10.16925/2357-5891.2018.02.06

\section{Resumen}

Objetivo: El emprendimiento se ha posicionado al interior de las universidades como uno de los temas importantes, por considerar que la formación disciplinar no es suficiente para desempeñarse exitosamente en el mundo actual; es necesario, además, formar capacidades y ofrecer alternativas de vida para los futuros profesionales con competencias en el siglo XXI. Los gobiernos también lo han vinculado dentro de sus agendas y han estructurado planes, programas y proyectos para fortalecerlo, entendiendo que su promoción puede contribuir al desarrollo económico y social de los países. El ensayo tiene como objetivo, demostrar la necesidad de fomentar políticas públicas en las universidades de América Latina, que tributen al emprendimiento para entregar un profesional con competencias en su incursión en el rol de empresarias en el desarrollo rural.

Metodología: Para ello, se han utilizados los métodos como el análisis síntesis, inducción deducción, histórico.

Resultados: En los últimos años, el fenómeno del emprendimiento ha aparecido de repente en nuestras sociedades como si se tratara de un movimiento cultural. Han aumentado las acciones, instituciones, unidades y servicios que se esfuerzan en apoyar el espíritu emprendedor.

Conclusiones: Es un hecho, que las universidades son una pieza fundamental en los ecosistemas de emprendimiento y de innovación.

Palabras claves: calidad, formación, fomento, modelo pedagógico. 


\title{
Entrepreneurship in rural women of the 21 st century
}

\begin{abstract}
Objective: the enterprise has positioned itself inside universities as one of the important themes, considering that disciplinary training is not enough to act successfully today; in addition, it is necessary to build capacities and offer life alternatives for future professionals with skills in the 21st century. Governments also link entrepreneurship within their agendas and structure plans, programs and projects to strengthen it, understanding that its promotion can contribute to the countries' economic and social development. This essay aims to demonstrate the need to promote public policies in universities in Latin America that tax the enterprise to train a professional with skills in his incursion into the role of business women in rural development.
\end{abstract}

Methodology: for this, methods such as synthesis analysis, induction, deduction, history were used.

Results: in recent years, the phenomenon of entrepreneurship has suddenly appeared in our societies as if it were a cultural movement. Actions, institutions, units and services that strive to support the entrepreneurial spirit have increased.

Conclusions: it is a fact that universities are a fundamental part of the entrepreneurial and innovation ecosystems.

Keywords: quality, training, promotion, pedagogical model.

\section{O empreendimento na mulher rural do século XXI}

\section{Resumo}

Objetivo: 0 empreendimento tem se posicionado no interior das universidades como um dos temas importantes, por considerar que a formação disciplinar não é suficiente para atuar com sucesso na atualidade; é necessário, além disso, formar capacidades e oferecer alternativas de vida para os futuros profissionais com competências no século XXI. Os governos também vinculam o empreendedorismo dentro de suas agendas e estruturam planos, programas e projetos para fortalecê-lo, entendendo que sua promoção pode contribuir para o desenvolvimento econômico e social dos países. Este ensaio tem como objetivo demonstrar a necessidade de fomentar políticas públicas nas universidades da América Latina que tributem para o empreendimento para formar um profissional com competências em sua incursão no papel de empresárias no desenvolvimento rural.

Metodologia: para isso, foram utilizados métodos como a análise síntese, indução dedução, histórico.

Resultados: nos últimos anos, o fenômeno do empreendimento tem aparecido de repente em nossas sociedades como se se tratasse de um movimento cultural. Têm aumentado as ações, instituições, unidades e serviços que se esforçam em apoiar o espírito empreendedor.

Conclusões: é um fato que as universidades são uma peça fundamental nos ecossistemas de empreendimento e de inovação.

Palavras-chaves: qualidade, formação, fomento, modelo pedagógico. 


\section{GLOSA INTRODUCTORIA}

En los últimos años, el fenómeno del emprendimiento ha aparecido de repente en nuestras sociedades como si se tratara de un movimiento cultural. Este, se reivindica con insistencia desde diferentes esferas y bajo un amplio rango de objetivos y propósitos. Han aumentado también las acciones, instituciones, unidades y servicios que se esfuerzan en apoyar el espíritu emprendedor. Es un hecho, que las universidades son una pieza fundamental en los ecosistemas de emprendimiento y de innovación. A pesar de ello, por su naturaleza, las universidades no destacan por una orientación demasiado emprendedora e innovadora. Por esta razón, a lo largo del tiempo las universidades se han enfrentado a importantes cambios estructurales y procesos de transformación en el desarrollo de sus principales actividades (docencia, investigación, y transferencia de conocimientos), los que requieren a su vez, la participación activa de diversos actores (gobierno, emprendedores/as, inversores/as, entre otros).

Desde esta perspectiva, la Administración Pública y la academia se han interesado por entender y recalcar el relevante papel de cada uno de los actores involucrados en la configuración de los ecosistemas de emprendimiento y de innovación. (Autio et al, 2014, pp. 1097-1108).

El ensayo tiene como objetivo, demostrar la necesidad de fomentar políticas públicas en las universidades de América Latina, que tributen al emprendimiento para entregar un profesional con competencias en su incursión en el rol de empresarias en el desarrollo rural. Para ello, se han utilizados los métodos como el análisis síntesis, inducción deducción, histórico,

\section{ENFOQUES, MODELOS, MECANISMOS Y ESTRATEGIAS DE INTERACCIÓN ENTRE LA UNIVERSIDAD-ENTORNO}

La universidad, como organización que tiene entre sus actividades la generación, difusión y transferencia de conocimientos, se ha convertido en un actor fundamental en la nueva economía, que entiende el conocimiento como un factor estratégico que genera ventajas competitivas para permitir la diferenciación de las organizaciones y su sostenibilidad en el contexto. Conscientes de ello, las universidades han empezado a dar mayor importancia a una de sus funciones sustantivas: la extensión universitaria o proyección social, mediante la cual esperan llevar conocimiento al entorno para contribuir al desarrolo local, regional, nacional e internacional.

En este sentido, la educación debe "fomentar la construcción de conocimiento (docencia) y la investigación científica (investigación) que generen impactos positivos en la sociedad, pero también debe permitir la identificación de oportunidades para el desarrollo regional o nacional y para el beneficio de la comunidad (extensión-proyección social)" (Ospina y Sanabria, 2010, p. 112).

Aunque la universidad surge alrededor de las escuelas monacales (escolástica), en los siglos XII y XIII, teniendo como referentes principales "la de París (1215) en Francia, la de Bolonia en Italia y la de Oxford en Inglaterra" (Restrepo, 1999, p. 19), la concepción de universidad moderna surge alrededor de 1810, con la creación de la Universidad de Berlín en Alemania, reconociendo entre sus funciones sustantivas la docencia e investigación.

En 1873, la Universidad de Cambridge, en Inglaterra, usó por primera vez el término extensión educativa, convirtiéndose en la tercera función sustantiva y en el elemento dinamizador que facilita el flujo continuo de conocimientos entre la universidad y la sociedad (Morales, Mira y Arias, 2010; Vega, 2002).

El propósito de la tercera función era la apertura de la universidad y su proyección social, tratando de responder a las necesidades de la comunidad. En estos inicios, las actividades de extensión se focalizaron en acercar la academia a la comunidad, a través de cursos de extensión en educación para adultos y capacitación para las comunidades circundantes, principalmente conferencias en agricultura (González y González, 2003; Torres, Sánchez y Márquez, 1996).

En América Latina, se comienza a hablar de la función social de la universidad a partir de 1918, cuando en el manifiesto del Movimiento de Córdoba, uno de los principios estaba dirigido al fortalecimiento de la función social de la misma a través de la tercera función sustantiva -la extensión-, vinculando la "universidad al pueblo" con el propósito de poner el saber universitario al servicio de la sociedad y hacer de sus problemas tema fundamental de sus preocupaciones (Rofman y Vázquez, 2006).

Por consiguiente, teniendo en cuenta que la universidad es una institución dinámica, Morales, Mira 
y Arias (2010) plantean que la función de extensión debe responder a diferentes contextos, haciendo frente a los procesos de globalización, democratización de la enseñanza y cambios políticos, para así estar en correspondencia con las demandas de la sociedad. En este sentido, dichos autores consultados identifican tres enfoques de extensión en los que vienen trabajando las universidades:

- Uno de corte cultural, cuyo fin es la transformación permanente de la cultura -saberes, conocimiento, habilidades, aptitudes y valores, arte, tecnología, experiencias- (Torres, Sánchez y Márquez, 1996, p. 6). Este busca la participación de todos en la misma cultura universitaria, acercando las comunidades. Esto implica que la universidad debe preservar, desarrollar y promover la cultura de la sociedad a través de la articulación de sus funciones sustantivas para corresponder a su cometido social (Vega, 2002).

- Otro de proyección social, que debe llevar a la academia, los estudiantes, los docentes y sus egresados a la búsqueda y solución de los problemas de la sociedad. Estos dos enfoques son propios de Latinoamérica y surgen como consecuencia de la Reforma de Córdoba que proponía conservar el carácter social de las instituciones universitarias (González y González, 2003; Torres, Sánchez y Márquez, 1996; Vega, 2002).

- El tercer enfoque está más vinculado con lo que se conoce como los conceptos universidad-empresa, universidad-industria o universidad empresarial, los cuales han sido propios de los países en desarrollo de economía neoliberal y de las universidades de Europa Occidental y Estados Unidos que, sin alejarse de muchos de los compromisos sociales (economía, cultura y responsabilidad social), han estado comprometidas principalmente con el desarrollo industrial y la prestación de servicios que correspondan con los cambios del entorno. Así, las universidades han sido parte fundamental en el desarrollo a nivel regional, primordialmente a través de la transferencia de tecnología y la venta de servicios públicos y privados (González y González, 2003; Vega, 2002).

Para ello, con la consecución adecuada de la relación academia-entorno, se han planteado distintos modelos teóricos tendientes a explicar la integración de las universidades con otros actores de la sociedad (Pineda, Morales y Ortiz, 2011). Lo anterior, teniendo en cuenta que el conocimiento es producido por diversos actores, como las universidades, los centros de desarrollo tecnológico, las empresas, los laboratorios estatales y privados, entre otros, y que son tres vértices los que principalmente estructuran el campo de generación y circulación de conocimiento: los sistemas políticos, los sistemas económicos y los sistemas educativos (Maldonado, 2008).

De acuerdo con Pineda, Morales y Ortiz (2011), las vinculaciones entre la academia y el entorno han sido abordadas desde modelos como el triángulo de Sábato y Botana (1986), el de la triple hélice (Etzkowitz y Leydesdorff, 1995) y el de sistemas de innovación (Lundvall, 1997), entre otros. Estos modelos, especialmente el de la "triple hélice", le asignan un rol protagónico a la universidad en la generación y transferencia de conocimiento e innovación hacia la sociedad. (Antúnez Sánchez y Díaz Ocampo, 2018)

Se justiprecia, como alrededor de dichas vinculaciones se encuentran apreciaciones que sustentan que mientras en los países desarrollados las contribuciones se concentran en el fortalecimiento de los vínculos universidad-industria a partir de la realización de investigaciones conjuntas, el desarrollo de patentes y la transferencia de tecnología (Tornatzky, Waugaman y Gray, 2002), en los países emergentes, los principales aportes de la universidad se orientan hacia la formación del capital humano, la adaptación de las innovaciones tecnológicas y su difusión en las empresas locales (Schiller, 2006).

De cualquier forma, lo que se evidencia es que, tanto en los países industrializados como en las economías emergentes, se están trabajando diversos mecanismos y estrategias que permitan llevar a cabo esta vinculación, aunque en su mayoría enfocados más a la relación de la universidad con el entorno empresarial (tercer enfoque de la extensión). En esta misma cuerda, se constata como existen diversos mecanismos y estrategias para la vinculación de la universidad con el entorno, los cuales pueden variar según el contexto de los países desarrollados y los países en desarrollo (Torres et al., 2009).

En el caso de los países desarrollados, se presenta una tendencia creciente de las corporaciones privadas a crear alianzas estratégicas con universidades, y, en muchos de ellos, se están introduciendo leyes sobre propiedad intelectual para favorecer la interacción. Los principales mecanismos y estrategias son las spin offs, spin outs y start ups las oficinas de transferencia de tecnología (OTRI), las incubadoras de empresas, 
los parques científicos y tecnológicos, las pasantías y prácticas profesionales, las consultorías, los programas de educación continuada, los centros de investigación cooperativa y de tecnología, las organizaciones regionales de innovación, las alianzas estratégicas o los consorcios, los centros o unidades empresariales y las políticas institucionales y nacionales, entre otros (Pineda, Morales y Ortiz, 2011).

En las economías en desarrollo como es el caso de las naciones latinoamericanas, son muy recientes y reducidos los esfuerzos para el establecimiento de una infraestructura física y social para el fortalecimiento de estas interacciones; parte de ello se evidencia en los escasos proyectos de investigación realizados en alianzas entre el sector productivo y académico, en la falta de claridad sobre el manejo de propiedad intelectual y en patentes o niveles de emprendimiento bajos, que en su mayoría son por necesidad y no por oportunidad (Pineda y Morales, 2010; Pineda, Morales y Ortiz, 2011).

Entre los mecanismos y las estrategias de interacción más comunes en la región están las pasantías o prácticas estudiantiles, además de los diplomados, las consultorías profesionales y las asesorías organizacionales brindadas por las universidades, a través de áreas administrativas con denominaciones cercanas a lo que podrían ser unidades de extensión. Más recientemente, se han promovido esfuerzos en el establecimiento de unidades de emprendimiento e incubadoras de empresas de base científica y tecnológica (Sutz, 2000).

Existe una gran cantidad de experiencias en las universidades latinoamericanas que muestran los diferentes caminos escogidos y de las que se pueden aprender valiosas lecciones, son los nuevos retos de la academia frente a la vinculación con su entorno. Es claro que los procesos de interacción universidad-sociedad deben ser el centro y la razón de ser de los programas de extensión.

Por ello, la reflexión sobre esta función sustantiva debe ser un proceso permanente y debe tener un reconocimiento y unos responsables dentro de la estructura de las universidades que permitan una planeación y una ejecución coordinada con las otras dos funciones: la docencia y la investigación, lo que permitirá una mayor pertinencia del quehacer de las instituciones de educación superior en su contribución a la solución de las diversas problemáticas de sus entornos (Ortiz y Morales, 2011, p. 361).

Otros retos en relación con lo anterior serian:
- Establecimiento de políticas y reglamentación de la extensión: la universidad debe establecer políticas y reglas de juego claras frente a la extensión (proyección social) de tal forma que sea evidente que está respondiendo a las necesidades de su contexto, sobre todo por su origen público y que, en el caso latinoamericano, se espera de ella no solo una respuesta hacia el sector productivo, sino también hacia la sociedad, desarrollando innovaciones sociales que involucren diversas instituciones y den solución a las problemáticas de la región (Morales, Pineda y Ávila, 2011). De igual forma, se requiere claridad sobre el manejo de la propiedad intelectual y la transferencia de conocimiento, de manera que estén acordes con la realidad que experimentan los investigadores colombianos y les permitan proteger y explotar sus desarrollos.

- Formación de capital humano competente: es necesario incentivar la formación permanente de docentes e investigadores en temas relacionados con la comercialización de ciencia y tecnología, capacitar facilitadores de vínculos con actores externos y estimular la formación en emprendimiento en los currículos académicos. Esto permitirá aumentar la acumulación de conocimiento e incentivar la producción intelectual requerida para la consolidación de una base científica que pueda ser llevada al entorno.

- Revisión y fortalecimiento de las pasantías universitarias: aunque existen beneficios para los actores involucrados, las críticas muestran que es necesario vincular el proceso de pasantías tanto a las dinámicas relativas al fortalecimiento de los planes de estudio de los diferentes programas como a los procesos de investigación aplicada que desarrollan los grupos de investigación, de manera que la universidad contribuya de forma efectiva a la solución de las problemáticas que se presentan en las diferentes organizaciones productivas, sociales y del Estado.

- Fortalecimiento de redes de apoyo: las redes de apoyo son un efectivo canal de comunicación entre los actores que es necesario consolidar si se desea que haya interacción dinámica entre los centros-grupos de investigación de la región (en las diferentes áreas del conocimiento) y las empresas, el Estado y los demás actores interesados. Establecer una infraestructura que favorezca estas interacciones requiere de la construcción de puentes entre el Gobierno, las universidades y las organizaciones de manera transdisciplinaria, para el 
aprovechamiento del capital humano y las capacidades científicas y tecnológicas existentes (Pineda y Morales, 2010).

- Generación de estructuras de soporte a la extensión: una acción fundamental para el adecuado desarrollo de la extensión es la generación de una cultura y estructura organizacionales que sustenten adecuadamente la función de extensión, a fin de evitar las limitaciones de enfoque que ya fueron señaladas.

Se reconoce, que la génesis de la enseñanza del emprendimiento tuvo su origen en los Estados Unidos de América con la labor de Miles Mace, con el primer curso de emprendimiento realizado en febrero de 1947, en la Escuela de Administración de Harvard. El propósito de dicho curso era el de capacitar a los ex combatientes de la Segunda Guerra Mundial para el mercado de trabajo, principalmente en lo concerniente a generación de autoempleo (Katz, 2003; citado en Henrique y Da Cunha, 2008).

En primera instancia, es importante reiterar que la función de extensión o proyección social no puede limitarse exclusivamente al desarrollo del emprendimiento o, aún peor, solo al emprendimiento empresarial, dadas las razones ya señaladas en el primer segmento. Así lo entienden algunas de las universidades más reconocidas en el país, en las que se considera al emprendimiento como uno más de los mecanismos de interrelación de la universidad con el entorno. Sin embargo, en esta parte del documento se reivindica al emprendimiento como una de las modalidades o campos de acción de la función de extensión cuyo papel ha sido reconocido como de gran importancia para las universidades y para la sociedad (Alvarado y Rivera, 2011).

Ahora, ¿Qué es el empredimiento? la palabra proviene del francés entrepreneur (pionero), y se refiere a la capacidad de una persona para hacer un esfuerzo adicional por alcanzar una meta u objetivo, siendo luego utilizada también para referirse a la persona que iniciaba una nueva empresa o proyecto, término que después fue aplicado a empresarios que fueron innovadores o agregaban valor a un producto o proceso ya existente y que actualmente se refiere además a la actitud que debe poseer una persona proactiva.

En este sentido, a continuación, se establecerán las razones de relevancia y la justificación del emprendimiento a partir de las tres funciones sustantivas de la universidad. En el desarrollo de este tema se establecen los conceptos fundamentales del emprendimiento. Por consiguiente, en los últimos años, se aprecia cómo se ha hecho evidente que las instituciones de educación superior (IES) han incorporado el tema del emprendimiento a sus procesos de formación, extensión e investigación, con diferentes énfasis, estrategias y acciones, debido a la importancia que se le ha dado en función del desarrollo económico y social. Los gobiernos también lo han vinculado dentro de sus agendas y han estructurado planes, programas y proyectos para fortalecerlo (Gibb y Hannon, 2007), entendiendo que su promoción puede contribuir al desarrollo económico y social de los países (Alvarado y Rivera, 2011; Cardona et al., 2008; Gámez, 2009; Rosa y Bowes, 1990, citados en Gómez y Satizábal, 2011).

En general, el fenómeno del emprendimiento puede entenderse como "la combinación entre la actitud y la capacidad de la persona, que le permiten llevar a cabo nuevos proyectos de cualquier índole, generalmente creativo. Se puede decir que el emprendimiento es la acción de convertir una idea (abstracta) en algo concreto (real)" (Morales, 2009). Por lo tanto, de forma amplia, el emprendimiento no necesariamente está relacionado con la creación de una organización o el beneficio económico; tal como lo señala Kunkel (1991, citado en Dehter, 2002). El ser emprendedor no está ligado a la idea de negocio lucrativo, ni a la innovación aplicada a la creación o mejora de bienes o servicios, ni al autoempleo o la satisfacción de necesidades económicas personales.

Lo anterior se esclarece completamente si se considera que el origen etimológico de la palabra emprendimiento se "deriva del término francés entrepreneur, que significa estar listo a tomar decisiones o a iniciar algo" (Rodríguez, 2009), pero puede volverse difusa si se considera la traducción del término inglés entrepreneurship pues, dada su ambigüedad, puede llegar a ser traducida como emprendimiento, empresarialidad, espíritu emprendedor, empresariado o incluso la de emprendedurismo (Gutiérrez, 2011).

El concepto de emprendimiento (entrepreneurship) designa la práctica de impulsar nuevas organizaciones, revitalizar organizaciones maduras e innovar creando nuevos negocios y proyectos en respuesta a la identificación de nuevas oportunidades. Incluye la creatividad, la innovación, la asunción de riesgos y la capacidad de planificar y gestionar proyectos. La competencia emprendedora incluye conocimientos, actitudes, habilidades, comportamientos y motivación. Algunos de estos atributos pueden ser inconscientes, otros innatos, mientras que otros se adquieren a lo largo del proceso de aprendizaje y formación. 
La inclusión de este concepto en la educación superior se ha visto justificada a partir de planteamientos como el de la Unesco (1998), que en su Declaración Mundial sobre la Educación Superior para el siglo XXI, donde se afirma que el rol de la universidad debe contemplar la necesidad de llevar los conocimientos al mundo del trabajo, y preocuparse por las acciones para "aprender a emprender y fomentar el espíritu de iniciativa”, con el fin de facilitar las condiciones de trabajo una vez concluida su formación profesional. De aquí su relación con el Derecho Laboral y con el Derecho Mercantil, lo cual parte de los textos constitucionales de cada nación, tras la firma de Convenios y Acuerdos internacionales.

Entonces, el reto para las universidades es la formación de individuos capaces de iniciar proyectos de distinta índole (económicos, políticos, públicos o sociales), de propiciar el cambio y el crecimiento de beneficios colectivos o, incluso, de asumir riesgos moderados y calculados como el que implica la creación de una empresa (Ecotec, 2007).

La formación, por tanto, deberá fundamentarse en motivadores diferentes a la necesidad, la supervivencia económica o la generación de riqueza, con proyección siempre hacia el futuro (Fontela, Guzmán, Pérez y Santos, 2006), pues la capacitación para los negocios y para la creación y administración de empresas no es suficiente para el desarrollo de las competencias propias de un emprendedor (Kirby, 2004). Esto habrá de ser uno de los principales diferenciadores entre el emprendimiento comercial y el emprendimiento social (Guzmán y Trujillo, 2008).

Se consideran entonces, como premisas fundamentales en la formación del emprendedor las siguientes habilidades a desarrollar y fomentar como: las técnicas (comunicación oral y escrita, trabajo en red), de gestión empresarial (planeación y conocimientos técnicos) y personales (asumir riesgos, innovación y constancia) (Hisrich, Peters y Shepherd, 2005; Kirby, 2004; Sarasvathy, 2001).

Por ello es que el autor consultado considera, El importante rol de las nuevas empresas creadas por egresados universitarios [...] es especialmente crucial en los países emergentes como Argentina (y América Latina en general) ya que la estructura industrial está compuesta por firmas de sectores tradicionales caracterizados por un relativamente bajo o moderado contenido tecnológico y que no juegan un rol importante como "organizaciones incubadoras" de nuevos emprendedores dinámicos (Kantis et al., 2001, p. 4).
El autor consultado realiza un estudio en Argentina, para identificar las diferencias existentes entre emprendedores universitarios y aquellos que no lo son. Encuentra que las empresas creadas por emprendedores de base universitaria se caracterizan por tener un perfil más calificado en cuanto a su mayor dinamismo, el talento humano que vinculan y el tipo de oportunidades que aprovechan, y porque aportan a la diversificación y el enriquecimiento de la industria; ello justifica la necesidad de promoverlas.

En este sentido, Lõbler (2006, citado por Toca, 2010) afirma que, en ambientes diversos, cambiantes e inciertos, los individuos deben formarse en emprendimiento, aprender a tomar buenas decisiones y a conducir iniciativas o proyectos, a generar sus propias respuestas frente a los acontecimientos del entorno, a dejar de buscar soluciones en experiencias pasadas y a aprender más sobre el saber que sobre el hacer.

También, Benegas y De Alto (2013) reflexionan en torno a la formación de ingenieros para una sociedad en transformación hacia la reindustrialización y el desarrollo tecnológico. Esta formación, requiere nuevos diseños curriculares, métodos pedagógicos y competencias por parte de los estudiantes. Por este motivo, es preciso incorporar nuevos ejes temáticos en el currículo: Ingeniería y conocimiento, innovación, empresarialidad, desarrollo local y desarrollo tecnológico. Consideran estos autores que, en la actualidad estos temas se desarrollan de manera fragmentaria, inconexa y a-sistémica. Con el objetivo de contribuir a generar en los futuros ingenieros una nueva cultura emprendedora e innovadora.

Se observa de esta manera, cómo se integra el emprendimiento en su desarrollo en tres funciones sustantivas de la universidad, se encuentra un énfasis en lo que compete a la formación: en la mayoría de instituciones se implementan y desarrollan programas, cursos y talleres de apoyo a los emprendedores, en algunos casos incorporados a la malla curricular, en otros, abiertos a los estudiantes que están interesados en el tema. Algunos críticos, sin embargo, advierten que en muchas instituciones los programas o cursos para emprendedores están más orientados a la elaboración de planes de negocio y seminarios sobre finanzas, marketing, marcos legales o descripciones del emprendedor, y que dejan de lado el desarrollo del capital social, es decir, los recursos individuales y las competencias específicas que permiten a las personas interactuar más efectivamente con otras. Estas cobran gran importancia para los emprendedores en 
aspectos como la selección de socios, la conformación de equipos y alianzas, el contacto con clientes e inversionistas, etc.

Donde a criterio del autor del ensayo cobra relevancia empoderarse de conocimientos desde las Ciencias de la Administración, en pos de la obtención de resultados positivos en la gestión como emprendedor. La investigación en el tema de emprendimiento también presenta diversos desarrollos y énfasis de acuerdo con los intereses particulares de los investigadores del fenómeno, con los avances de los diversos grupos de investigación que se han conformado en torno al tema o con la importancia que le han otorgado las diferentes instituciones al emprendimiento. Actualmente se constata que se están diseñando y poniendo en marcha acciones concretas para el fomento del emprendimiento en los estudios universitarios, si bien existe una visión bastante restringida del término emprendimiento, pues se asimila a «crear empresas» $\mathrm{o}$ «montar tu propio negocio».

En este sentido, se confirma la generalización de un punto de vista comercial y estrictamente mercantilista del término, sin que haya muchas opciones de transferencia a otros contextos (ámbito social, educativo, sanitario, etc.), dado que no está muy extendida ni aceptada una visión amplia del emprendimiento, aquella que podría asociarse a competencias o actitudes emprendedoras como, por ejemplo, tener iniciativa personal y capacidad para asumir riesgos y responsabilidades o para trabajar en equipo.

En los diferentes eventos académicos, en la región de Latinoamérica se puede evidenciar que el tema se constituye como un nuevo eje temático en torno al cual se reúnen un buen número de investigadores con diversas propuestas, metodologías y resultados que pretenden ampliar el cuerpo de conocimientos que existe sobre el emprendimiento. De esta forma, "el emprendimiento como temática de estudio de las ciencias económicas y de la gestión fue creciendo hasta configurar, sino un campo de investigación, al menos un interés emergente entre los investigadores que tratan de precisar las características de este fenómeno y en especial de analizar su estatus epistemológico" (Alvarado y Rivera, 2011, p. 63).

En tal sentido, puede considerarse que aún no hay una base de datos que agrupe los estudios e investigaciones que se han llevado a cabo en otras plataformas digitales, como es la plataforma Scienti de Colciencias, ahora sí se puede identificar que los grupos interesados en el tema han aumentado en la última década. En la actualidad, se pueden identificar nueve grupos visibles, aunque es posible encontrar estudios referentes al tema en otros grupos. Al realizarse una agrupación preliminar de los estudios llevados a cabo por los diferentes grupos de investigación, se pueden encontrar las siguientes categorías:

Formación: proyectos relacionados con los procesos de formación (cátedras y otras actividades) que adelantan las instituciones educativas. Se mide también con alguna frecuencia la actividad emprendedora de los egresados de cada universidad.

Incubación: proyectos relacionados con creaciones de centros o entes encargados de apoyar la creación de empresas.

Pymes: proyectos que abordan diversas problemáticas de la pequeña y la mediana empresa desde la perspectiva del emprendimiento.

Empresarios: proyectos realizados teniendo como eje la historia empresarial colombiana o perfiles empresariales actuales.

Oportunidades: proyectos relacionados con la identificación de ideas de negocio.

Sectores: estudios que dan cuenta de la problemática específica de empresas y empresarios en algunos sectores económicos.

Finanzas: proyectos centrados en aspectos financieros de las empresas o los emprendedores.

Esta clasificación, muy general, permite identificar las tendencias y los intereses de los académicos que desean contribuir a generar conocimientos sobre el tema del emprendimiento.

Respecto a la función de extensión, el emprendimiento se liga con la visión actual de que la universidad es un agente de transferencia de conocimiento y de tecnología, y de que la base de este proceso son la innovación y el emprendimiento, teniendo siempre como fin último el compromiso social con el entorno en el que actúa e influye.

En este sentido, Bueno, Casani y Lizcano (1999), plantean que esta visión de la extensión se enmarca en el conjunto de actividades que las universidades llevan a cabo con los diferentes agentes sociales con los que se relacionan y a los que transfieren su conocimiento; en la transferencia de I+D que, en suma, genera la innovación que la sociedad reclama; en los esfuerzos que responden al compromiso social que tienen con su entorno como servicio público y en la generación de ingresos adicionales, aunque este no sea el objetivo último. Estos ingresos se derivan 
del proceso de comercialización tecnológica de los recursos universitarios.

Plantean los autores consultados, que esto responde a la visión proactiva de la universidad para llevar a cabo acciones de desarrollo tecnológico, asistencia técnica, programas de formación continua, programas de posgrado y contratos de investigación, entre otras actividades, con corporaciones, fundaciones, empresas, entidades gubernamentales, asociaciones, etc. Se propone, entonces, una "tercera misión" de la universidad, a través de su actividad emprendedora, es decir, una misión construida visualizando una universidad para la transferencia de I+D o del conocimiento tecno-científico.

Como ocurre con la mayoría de aspectos de la realidad, el emprendimiento no se genera de forma espontánea y aislada, sino que se origina y depende de otros aspectos. Por tanto, para la comprensión y potenciación del emprendimiento se requiere de un conocimiento de los demás factores que lo afectan, es decir, la comprensión de aquellos aspectos que constituyen lo que se puede denominar la estructura para el emprendimiento.

En este apartado se van a evidenciar las variables que son consideradas como de mayor importancia para el fenómeno del emprendimiento y que, por supuesto, deberían ser tenidas en cuenta en el desarrollo de cualquier iniciativa de generación o fortalecimiento de este tipo de programas, ya sea en el entorno universitario, en el ámbito normativo o en el contexto organizacional. En cuanto a la estructura requerida para el emprendimiento, es claro que en ella intervienen tanto factores económicos como aspectos sociales, culturales, políticos, ambientales, tecnológicos, etc. Por lo tanto, sin una adecuada estructura para el emprendimiento, cualquier esfuerzo que se realice para fomentarlo se constituirá en una acción aislada y con poca probabilidad de éxito.

Así, el emprendimiento requiere de la construcción de un ambiente propicio, que se encuentre más allá de las buenas intenciones y los propósitos gubernamentales, organizacionales o individuales, y de fines coyunturales, y que lleva a transitar hacia la intervención de los verdaderos factores que pueden limitar o impedir su adecuado desarrollo (aspectos estructurales). Kunkel (1991, citado en Dehter, 2002) considera que estos factores intervinientes pueden ser determinados por tres actores fundamentales: el Estado, las instituciones de educación superior y las empresas.
También es fundamental la formación del profesorado universitario. Se requiere una formación encaminada a conocer, trabajar y poner en práctica las diferentes competencias emprendedoras. Una formación transversal que realice una primera aproximación a este conjunto de competencias, la cual podría llevarse a cabo desde instancias institucionales. Una formación básicamente de carácter práctico y que sobre todo oriente el aparato metodológico y de estrategias concretas para trabajar el emprendimiento en las aulas universitarias.

En cuanto a las empresas como sujetos de gestión económica, las variables sobre las que pueden intervenir para facilitar el desarrollo del emprendimiento son: fomentar el intraemprendimiento, la eliminación de los esquemas jerárquicos, el favorecimiento del comportamiento emprendedor, la creación de cultura de emprendimiento, la transmisión del emprendimiento a las asociaciones empresariales y profesionales, la promoción de las fortalezas de los intraemprendedores y la generación de sistemas de incentivos para retener a la gente creativa.

Por su parte, a partir de varios autores, Vesga ha planteado la existencia de tres niveles interactuantes que determinan el desarrollo del emprendimiento innovador:

En el nivel macro establece los componentes del entorno que afectan favorablemente a las organizaciones e individuos para emprender, a saber: a) mayor grado de producto per cápita, b) mayor tamaño del mercado, c) libertad para competir y mayores garantías para las empresas pequeñas, d) el uso de tecnologías y e) fluctuaciones favorables en las variables demográficas.

En el nivel de las empresas, este autor consultado establece como variables que favorecen el emprendimiento las siguientes: a) la determinación de la estrategia y el compromiso con ella, b) el aseguramiento de los recursos y las capacidades para generar ventajas sostenibles, c) una cultura organizacional abierta, con jerarquías planas y multidisciplinares, y compromiso de la dirección con la innovación, y d) decisiones alineadas con los intereses de largo plazo de la organización. Paralelamente, en este nivel están las universidades que pueden favorecer el emprendimiento en: a) la producción del conocimiento, b) la difusión del conocimiento y c) la aplicación del conocimiento.

En el tercer nivel, el de los individuos, el emprendimiento se favorece a través de los siguientes 
aspectos: a) el alto nivel educativo, b) el desarrollo de habilidades venidas de la experiencia, c) el desarrollo de la capacidad de identificar oportunidades y atender necesidades, d) la disposición para el riesgo con la promesa de ganancias y e) la existencia de las cualidades o los recursos psicológicos requeridos (creatividad, perseverancia, iniciativa, autonomía, motivación, entre otras).

Este autor consultado, establece que las variables que pueden intervenirse para afectar las variables ya mencionadas son de dos tipos: las que conectan el nivel macro y el empresarial (instituciones, políticas gubernamentales y reglas de juego; infraestructura del país; acceso a capital para crecimiento; conocimiento sobre operación de la innovación y pertenencia a redes de este tipo; efectividad de la relación universidad-empresa) y variables que conectan el nivel macro y el de los individuos (instituciones y reglas de juego para la creación de empresas, acceso a capital para creación de empresas, incentivos para el comportamiento innovador, reducción del riesgo mediante la disponibilidad de información sobre oportunidades y pertenencia a redes que ayuden a superar las barreras)

Por ello, en el ensayo se colige que emprender en el Ecuador no es igual que hacerlo en países denominados del primer mundo, tampoco es lo mismo si lo hace una persona que terminó el colegio comparada a otra que terminó la universidad o que posee amplios conocimientos de cursos de maestrías. Hay otros elementos a ponderarse como el dominio de ciertas herramientas básicas como la contabilidad, las finanzas y el mercadeo; pero que, aun teniendo pleno conocimiento de estas herramientas, ningún emprendedor debe pensar que sus planes de negocio tienen garantizado su éxito.

En definitiva, la creatividad, el talento, el tesón e inclusive la suerte que hay que tener para lograr una recompensa económica sostenida a lo largo del tiempo pueden ser saboteadas por factores externos al emprendimiento: cambios en las políticas económicas, nuevas reglas institucionales de juego, incertidumbre jurídica, etc., los que entorpecen el proceso de la planificación empresarial.

Otro elemento de peso a ponderarse, está en focalizado en el hábitat natural del emprendedor con la economía de libre mercado en el marco de un fuerte Estado de Derecho. Comprendiendo por Estado de Derecho al ordenamiento institucional (reglas del juego) en donde impera la ley y en donde el poder político está constitucionalmente limitado a garantizar los derechos a la vida, propiedad y libertad.

Por ello, un emprendedor del siglo XXI será aquel que promueva la libertad económica para todo el mercado, no solo para su ámbito de negocio. Entenderá que la libertad económica es un principio universal. Comprenderá que el mercado libre implica un trato voluntario, moral y pacífico entre hombres iguales que se respetan e intercambian sus derechos de propiedad sobre bienes y servicios.

Son todos estos elementos abordados los que permitirán realizar un acercamiento al comportamiento de las políticas públicas relacionadas con la mujer rural en América Latina, en el emprendimiento agropecuario en el rol de pequeña empresaria.

\section{LA MUJER RURAL, DESAFÍOS EN EL SIGLO XXI EN EL EMPRENDIMIENTO AGROPECUARIO COMO PEQUEÑA}

\section{EMPRESARIA}

Como suceso histórico, social, político y económico, América Latina y el Caribe se presenta, ya entrado el siglo XXI, como un continente contradictorio y heterogéneo, en el que conviven realidades de profundas diferencias. A la vez que se destacan en el continente las llamadas economías emergentes por sus años sostenidos de crecimiento económico, perduran casos de persistente pobreza y precariedad social e institucional. Ello permite confirmar que la participación de la mujer en la fuerza laboral ha aumentado en forma sostenida en las últimas décadas y al mismo tiempo se ha ido acortando la brecha de género en el empleo, ésta sigue siendo considerable.

Se aprecia en las reformas realizadas, las que incluyen, entre otros, cambios constitucionales, creación de ministerios o institutos para los asuntos de la mujer, modificaciones al código civil, penalización de la violencia de género y la adopción de políticas que apuntan a la equidad de género. En el ámbito político también se ha progresado de manera estable, aunque la proporción de mujeres en los gobiernos locales y nacionales sigue estando por debajo del $50 \%$ en la mayoría de las naciones.

El emprendimiento es considerado como la capacidad de diseñar una idea, identificar las oportunidades y plasmarlas en un proyecto (Sierra, 2014). Es así que la búsqueda de oportunidad se vuelve 
imprescindible, siendo el motor que impulsa a viabilizar la idea, generando recursos, opciones y el alcance necesario para lograr el éxito empresarial.

En el emprendimiento femenino, las responsabilidades familiares siguen siendo una importante limitación en América Latina ya que aún persiste la tradicional división de roles, donde el hombre es el proveedor y sostén del hogar y la mujer la administradora del hogar y la responsable de la crianza de los hijos (Hernández, Camarena y Castanedo, 2009).

Sin embargo, Escandón y Arias (2011) señalan que en los últimos años existe una tendencia creciente de las mujeres a cambiar estos roles, por ejemplo, asumiendo el rol de empresaria, disminuyendo la brecha existente con los hombres y abriéndose paso hacia mayores posibilidades de educación, al desarrollo de capacidades y conocimientos y a la mayor incorporación al mercado laboral.

Entonces, es un hecho que, los emprendimientos en su inmensa mayoría no se han enfocado con una mirada de género, incluye en la misma acepción diferentes niveles de desarrollo y complejidad de ese trabajo, el que puede incluir tanto a personas autoempleadas como a aquellas que tienen una empresa mediana o pequeña para la cual necesitan contratar fuerza laboral, donde el Derecho Mercantil traza las pautas jurídicas en este sentido.

Aunque, en pleno siglo XXI no se pueda hablar de total equidad en el entorno laboral para las féminas. $\mathrm{Al}$ persistir brechas que inciden en la no articulación mejor de las variables territorio, género y juventud; para profundizar en los factores específicos que en las localidades pueden estar determinando las brechas de género y el rezago que experimentan las mujeres rurales, entre ellas las jóvenes, en su empoderamiento.

Se parte al considerarse que un emprendedor como aquella persona que convierte una idea en un proyecto concreto, lo puntualiza y organiza los recursos que se necesitan para su desarrollo y para convertirlo en una realidad que produzca beneficios. Este tiene conocimientos solidos de la actividad en la que se desarrolla y posee habilidades de proactividad ante los cambios actuales, en estado de incertidumbre y volatilidad, tanto en los ámbitos microeconómicos como macroeconómicos.

Así pues, SELA (2010) señala que las características de las mujeres emprendedoras en América Latina son muy marcadas y estas son:

- El motor detrás de la empresa de mujeres, como regla general, está en la necesidad económica más que en la identificación de una oportunidad o en un deseo de desarrollar una idea innovadora.

- Las mujeres son dueñas de empresas más pequeñas que las empresas de los hombres.

- El nivel de educación de las mujeres empresarias en ALC en general es más bajo que el de los hombres.

- Las empresas de mujeres en ALC operan de manera predominante en el sector servicios y están mejor posicionadas para atender nichos de mercado.

- Las mujeres empresarias enfrentan dificultades estructurales para acceder a crédito y capital limitado, lo que impide que sus empresas crezcan al tiempo que carecen de acceso a educación financiera.

- La conciliación entre vida familiar y empleo se resuelve muchas veces a través de una

- empresa, pero al mismo tiempo hace también difícil desarrollar la empresa.

- Las mujeres empresarias enfrentan estereotipos negativos sobre su capacidad para establecer y desarrollar una empresa.

El hecho del empoderamiento de las mujeres rurales en América Latina y el Caribe, ante la limitante de por la condición de las mujeres por su condición de género es particular. Las mujeres continúan teniendo menor acceso a la tenencia de la tierra, menores ingresos, discriminación de género, como acceso a la seguridad social. En otras palabras, aún no poseen los derechos de propiedad y titularidad. Perdurando brechas de desigualdad en este sentido para con los hombres. El estudio realizado por la FAO (2011) así lo demuestra.

Ahora, hacia donde van las políticas públicas en este sentido, toda vez que hay limitantes en cuanto al acceso a la tierra, al uso de las tecnologías y de los mercados que faciliten el empoderamiento a la mujer rural (mujeres indígenas y las afrodescendientes) como parte del reconocimiento cultural al señalarlos los textos constitucionales con el pluralismo jurídico, ante los adversos generados por el cambio climático, la problemática de la seguridad alimentaria en la región, la crisis de los precios como temas centrales en la agenda de desarrollo del bloque latinoamericano, que permita transformar el actual estatuto agrícola promovidos por el Ministerio Agrícola y el de Trabajo respectivamente. Que permita con ello la dignificación de la mujer rural en América Latina. (Antúnez Sánchez, Díaz Ocampo, 2017)

Demostrándose que se incumple con la Convención sobre la eliminación de todas las formas de 
discriminación contra la mujer, firmado en New York en el 2000. Que permita lograr en la región cumplir los principios de igualdad, convivencia, tolerancia e inclusión, la equidad de género que enfatiza la búsqueda de equilibrio entre hombres y mujeres en derechos, espacios y oportunidades. Constituye la principal política pública para promover el desarrollo integral de la mujer y su participación en todos los niveles de la vida económica, política y social.

Sus principales ejes son la promoción, el desarrollo y el respeto a los derechos humanos individuales, colectivos, económicos, sociales, políticos, culturales y ambientales de las mujeres. Este es uno de los paradigmas dentro del Derecho Agrario, el desarrollo rural. Se materializa la implementación de las ideas de los gobiernos regionales y locales en torno a mayores atribuciones, competencias y recursos, ello será el factor de despegue en los procesos productivos en Latinoamérica, donde la mujer rural se empodera. Que falta, mayor autonomía y capacidad en materia conductiva, el impulso de políticas territoriales, y la articulación de acciones y procesos donde se incluya más al ciudadano.

Los aportes que, desde las Ciencias de la Administración, se constata como la cultura organizacional influye en las características de un emprendedor, aunque haya características generales que pueden identificarlos, como seguridad, adopción de riesgos, independencia, visión, creatividad, entre otras. Sin embargo, como puede apreciarse, se trata de condiciones de las que pueden disfrutar también, en muchos casos, personas no emprendedoras, por lo que definir un perfil general para los mismos, resulta complicado. Inclusive, se puede constatar la existencia de empresarios exitosos que no se consideran como tales, emprendedores, como podría ser en el caso de la herencia de un negocio.

Otro elemento a tenerse en cuenta es el liderazgo, influye en las funciones de la cultura (integración interna, adaptación externa) y tiene un rol fundamental en la formación, consolidación y cambio cultural y de ningún modo puede subestimarse su papel. Un líder debe comunicar con claridad a sus colaboradores y seguidores sus valores, visión, misión, objetivos, estrategia, propuesta y programa de trabajo para alinear los esfuerzos en una misma dirección. Deben ser, agentes de innovación y cambio que guían, desarrollan y hacen crecer a sus organizaciones o grupos. Un líder de ser innovador y creativo, idear nuevas soluciones, desafiar la forma en que se hacen las cosas y proponer hacer cosas distintas, es importante y necesario ante un mundo muy competitivo, que avanza rápidamente en el contexto de un cambio tecnológico sin precedentes, que necesitan comprender en su justa dimensión y analizar y sintetizar eficazmente la información que reciben.

Esta reconocido por los estudiosos del tema, que el nivel educativo no es el único obstáculo que enfrentan las mujeres emprendedoras, pues diversos estudios (Powers y Magnoni, 2010, Heller, 2010) señalan que el mayor desafío es que no cuentan con experiencia empresarial y en comercialización, así como la insuficiente formación empresarial específica (administración/marketing). Con respecto a la falta de experiencia, Díaz y Jiménez (2010) señalan que se debe generalmente a que las empresarias crean su empresa cuando son más jóvenes y adquieren experiencia en su propia empresa y no como empleadas asalariadas. Esto las pone en desventaja, pues de acuerdo con la OCDE (2012) la experiencia es crítica para el éxito de cualquier negocio. Corroborándose esto con lo que señalan Escandón y Arias (2011) y SELA (2010), que las empresas dirigidas por mujeres con más educación son las que logran entrar al sector formal, así como una mayor expansión en el mercado siendo más competitivas, lo cual se refleja en mayor productividad para estas.

De hecho, se puede apreciar como las mujeres empresarias se concentran en la pequeña y microempresa. Esta diferencia puede explicarse por el vínculo que existe entre desigualdades de género y la propiedad de los bienes productivos, como la tierra y el capital, y su impacto negativo sobre la capacidad de la mujer de aprovechar las oportunidades que trae consigo el desarrollo económico.

Las empresarias de América Latina y el Caribe, se encuentran focalizadas sobre todo en la pequeña y la microempresa y muy pocas están en empresas grandes o medianas. Los negocios encabezados por una mujer también suelen concentrarse en un menor número de sectores económicos (comercio, servicios y manufactura) que las empresas de propiedad de hombres. La región tiene la tasa más alta de emprendimiento femenino y pervive la brecha entre hombres y mujeres empresarias, la que es menor que en otras regiones del planeta.

Con ello se demuestra que hay aun desigualdad en la sociedad latinoamericana, focalizados en la posesión de la tierra, las limitantes en relación con el acceso a los créditos financieros, con el acceso a obtener tecnologías de avanzada en materia agropecuaria, 
son elementos que inciden en la invisibilidad de la mujer rural, al no reconocerse su valor productivo ante la multiplicidad de roles que esta desempeña, los que impiden la feminización agrícola.

\section{Corolario}

- En los últimos años, las universidades han implementado nuevos modelos para hacer frente a las demandas sociales, debido a las elevadas tasas de desempleo juvenil, a la reducción de los presupuestos en educación y al aumento de la competencia de los sujetos de gestión. Ante esta situación, las universidades, que a su vez forman parte de sus respectivos ecosistemas de emprendimiento e innovación, requieren de una actitud proactiva y de una interconexión constante con sus stakeholders.

- En América Latina, a pesar de los avances observados en ambos ecosistemas, no hay evidencia suficiente para reconocer que es hay una sociedad emprendedora y que cuyo crecimiento, competitividad y sostenibilidad esté relacionado con las iniciativas emprendedoras. En este sentido, uno de los principales retos sería la transformación de la sociedad actual hacia una sociedad más emprendedora.

- Hay que revertir la situación del hombre rural como agricultor y la mujer como ayudante, las políticas públicas en la región de América Latina deberán estar dirigidas en esta dirección para con ello revertir la historia tradicional que desde el pasado siglo XX ha estado imperando con un nuevo enfoque de género en pos de alcanzar el desarrollo sostenible.

- Las ONG y los organismos regionales tiene un valor inestimable en sus estudios en la región, al demostrar la necesidad de modificar el status actual de la mujer rural en América Latina, lo demuestra los análisis realizados por la FAO, MERCOSUR, CEPAL, y CELAC, entre otras.

- El emprendedurismo es considerado cada vez un factor más importante para contribuir al desarrollo económico, la generación de puestos de trabajo, la inclusión social, la reducción de la pobreza y el desarrollo de la innovación. La educación universitaria cumple un rol fundamental en el proceso de desarrollo emprendedor.

\section{REFERENCIAS}

AA. VV. (2014) Estado del arte sobre emprendimiento universitario, Ministerio de Comercio, Industria y Turismo y Asociación Colombiana de Universidades.

AA. VV. (2010) Mujeres empresarias en America Latina, Programa de Promoción de Equidad de Género y Derechos de la Mujer, Editorial LEDEL SAC, Peru.

AA. VV. Ecuador ¿País de Emprendedores? Cámara de Comercio de Guayaquil y la Universidad Ecotec, Ecuador.

AA. VV (2013) Enfoque territorial para el empoderamiento de las mujeres Rurales en America Latina y el Caribe, ONU

AA. VV. (2010) Jovenes emprendedores rurales: Una aproximación a la evolución y estado actual de los servicios de apoyo en América Latina, Programa de Talentos Rurales, Argentina.

AA. VV. (2017) El emprendimiento y el socialismo del siglo XXI en el Ecuador: ¿Una vinculación paradójica o un apoyo al desarrollo económico?, Revista Dilemas Contemporáneos: Educación, Política y Valores,

Ajzen, I. (1991). The theory of planned behavior. Organizational Behavior and Human Decision Processes, 50, 179-211.

Alvarado, O. y Rivera, W. F. (2011). Universidad y emprendimiento, aportes para la formación de profesionales emprendedores. Cuadernos de Administración (45), 61-74.

Álvarez C. y Urbano D. (2011). Environmental factors and entrepreneurial activity in Latin America. Revista Latinoamericana de Administración-Cladea, 48, 31-45.

Alemany, L., Álvarez, C., Planellas, M y Urbano, D. (2011) Libro blanco de la cultura emprendedora en España. Fundación Príncipe de Girona. ESADE. Barcelona.

Ararat, J. (2010). La ideología del emprendimiento. Una mirada desde el análisis crítico del discurso. Revista Administer, 17, 5-33.

Autio, E.; Kenney, M.; Mustar, P.; Siegel, D.; Wright, M. (2014). Entrepreneurial innovation: The importance of context. Research Policy, 7, 1097-1108.

Briceño, M. (2009). La innovación como accionante del emprendimiento en las organizaciones. Borradores de Administración, 31, 1-21.

Bueno, E., Casani, F. y Lizcano, J. (1999). Formación de la estrategia empresarial: un análisis de las dinámicas del proceso estrategia. Revista Española de Financiación y Contabilidad, 100, 195-217. 
Benegas, M; De Alto, B. (2013) Exi2 - Espacio, ingeniería e innovación. Ponencia presentada en: Formando comunidades para el emprendimiento sustentable: VII Workshop Red Emprendesur. Medellín.

Briasco, I. (2014) El desafío de emprender en el siglo XXI, Paseo Imperial, España.

Cardona A. M., Vera A. L. y Tabares Quiroz, J. (2008). Las dimensiones del emprendimiento empresarial: la experiencia de los programas cultura y fondo emprender en Medellín. Cuadernos de investigación (69), 64.

Capelleras, J. y Kantis, H. (2009) Nuevas empresas en América Latina: factores que favorecen su rápido crecimiento. Universidad Autónoma de Barcelona. España.

Christensen, S. (2005). Enabling intrapreneurship: the case of a knowledge-intensive industrial company. European Journal of Innovation Management, 8 (3), 305318.

Dehter, M. (2002). El ser emprendedor: modelo para desarrollar comportamiento emprendedor individual y corporativo. Recuperado el 3 de enero de 2014 de: https:// www.u-cursos.cl/ingenieria/2005/1/IN505/1/buscador/?q=ser+emprendedor.

Ecotec (2007). Design and implementation of the pilot action "ERASMUS for young entrepreneurs". Enterprise and industry. Working Paper, 2.

Etzkowitz, H. (2002). The Triple Helix of University-Industry-Government Implications for Policy and Evaluation. Working Paper Science Policy Institute, 11.

Etzkowitz, H. (2004). The evolution of the entrepreneurial university. International Journal of Technology and Globalisation, 1 (1), 64 - 77.

Etzkowitz, H. y Leydesdorff, L. (1995). The Triple Helix University Industry Gobernment Relations: A laboratory for Knowledge based Economic Development. EASST Review, 14, 14-19.

Fontela, E., Guzmán, J., Pérez, M. y Santos, F. (2006). The art of entrepreneurial foresight. Madrid: Universidad Antonio de Nebrija.
Formichella, M. (2004). El concepto de emprendimiento y su relación con la educación, el empleo y el desarrollo local. Buenos Aires: Instituto Nacional de Tecnología Agropecuaria.

Henrique, D. \& Da Cunha, S. (2008) Práticas didático-pedagógicas no ensino de empreend-edorismo em cursos de graduação e pós-graduação nacionais e internacionais. Administração Mackenzie, 9(5) 112-136.

Heller, L. Mujeres emprendedoras en América Latina y el Caribe: realidades, obstáculos y desafíos. Mujer y Desarrollo, No 93. CEPAL. Santiago de Chile. 2010.

Hamburger, Á. (2014) El socialismo del siglo XXI en América Latina: Características, desarrollos y desafíos. Revista de Relaciones Internacionales, Estrategia y Seguridad, 9(1), 131-154.

Gámez, J. (2009). Aproximación a los modelos de emprendimiento. Management, 18 (31), 153-170.

Garcia Arango, G. Tesis doctoral: La regulación jurídica del emprendimiento en Latinoamérica y España, Universidad de Alicante, España (2017)

González, A. (2011) Aprender a emprender. Extoicos. 2: 108 $-110$

Guerrero, M. y Urbano, D. (2014) Emprendimiento e innovación: realidades y retos de las universidades españolas: Ministerio de Economía y Competitividad de España.

Parra, R; Mesa, J.H; Correal, S. (2009) La historia del empresarismo en EAFIT. Revista Universidad EAFIT. 45 (154) $78-97$

Sparano, H. (2014) Emprendimiento en América Latina y su impacto en la gestión de proyectos. Revista Dimensión Empresarial, №. 12, pp. 95-106.

Moulden, J. (2009) Los nuevos emprendedores sociales, Editorial McGraw Hill Interamericana, México. 